\title{
MRI Ultrasound Fusion Guided Biopsy
}

National Cancer Institute

\section{Source}

National Cancer Institute. MRI Ultrasound Fusion Guided Biopsy. NCI Thesaurus. Code

C118510.

A method for targeted biopsy that merges images captured by multiparametric MRI with real-time ultrasound, creating a synthetic, 3D image, which highlights the suspicious area within the image. 